\title{
A reduction technique for Generalised Riccati Difference Equations arising in linear-quadratic optimal control
}

\author{
Augusto Ferrante and Lorenzo Ntogramatzidis
}

\begin{abstract}
In this paper we develop a reduction technique for the generalised Riccati difference equation arising in optimal control and optimal filtering. This technique relies on a decomposition method for the generalised Riccati difference equation that isolates its nilpotent part, which becomes constant in a number of iteration steps equal to the nilpotency index of the closed-loop, from another part that can be computed by iterating a reduced-order Riccati difference equation.
\end{abstract}

\section{INTRODUCTION}

In this paper we are concerned with the solutions $X_{t}, t \in$ $\{0, \ldots, T-1\}$ of the so-called Generalised Riccati Difference Equation $\operatorname{GRDE}(\Sigma)^{1}$

$$
\begin{gathered}
X_{t}=A^{\mathrm{T}} X_{t+1} A-\left(A^{\mathrm{T}} X_{t+1} B+S\right)\left(R+B^{\mathrm{T}} X_{t+1} B\right)^{\dagger} \\
\cdot\left(B^{\mathrm{T}} X_{t+1} A+S^{\mathrm{T}}\right)+Q
\end{gathered}
$$

iterated backwards from $t=T-1$ to $t=0$ using the terminal condition

$$
X_{T}=P,
$$

where $T>0, A \in \mathbb{R}^{n \times n}, B \in \mathbb{R}^{n \times m}$, with $m \leq n$; the matrices $Q \in \mathbb{R}^{n \times n}, S \in \mathbb{R}^{n \times m}$ and $R \in \mathbb{R}^{m \times m}$ are such that the socalled Popov matrix

$$
\Pi \triangleq\left[\begin{array}{cc}
Q & S \\
S^{\top} & R
\end{array}\right]
$$

is symmetric and positive semidefinite. Finally, the terminal condition $P \in \mathbb{R}^{n \times n}$ is assumed to be symmetric and positive semidefinite. Despite the fact that it has been known for several decades that the generalised discrete Riccati difference equation provides the solution of the classic finitehorizon LQ problem under the weakest system-theoretic assumptions on the Popov triple [10], this equation has not been studied with the same attention and thoroughness that has undergone the study of the standard discrete Riccati difference equation (in which the Moore-Penrose pseudoinverse is replaced by the standard matrix inverse). The purpose of this paper is to present a reduction technique

Partially supported by the Italian Ministry for Education and Research (MIUR) under PRIN grant n. 20085FFJ2Z "New Algorithms and Applications of System Identification and Adaptive Control" and by the Australian Research Council under the grant FT120100604.

A. Ferrante is with Dipartimento di Ingegneria dell'Informazione, Università di Padova, via Gradenigo, 6/B - 35131 Padova, Italy. augusto@dei.unipd.it

L. Ntogramatzidis is with the Department of Mathematics and Statistics, Curtin University, Perth (WA), Australia. L. Ntogramatzidis@curtin.edu.au

${ }^{1}$ The symbol $M^{\dagger}$ denotes the Moore-Penrose pseudo-inverse of matrix $M$. for this equation that allows to compute its solution by solving a smaller equation with the same recursive structure, with obvious computational advantages. In order to carry out this task, several other important side results on the corresponding generalised Riccati equation are established, which constitute an extension of those valid for standard discrete algebraic Riccati equations presented in [7] and [2]. In particular, these results show that the nilpotent part of the closed-loop matrix is independent of the solution of the generalised algebraic Riccati equation. Moreover, we provide a necessary and sufficient condition expressed in sole terms of the problem data for the existence of this nilpotent part of the closed-loop matrix. This condition, which appears to be straightforward for the standard algebraic Riccati equation, becomes more involved - and interesting - for the case of the generalised Riccati equation.

The $\operatorname{GRDE}(\Sigma)$ with the terminal condition (2) will be referred to as the Generalised Riccati Difference Problem $\operatorname{GRDP}(\Sigma, P)$. An important observation is that all matrices of the sequence $\left\{X_{t}\right\}_{t=0, \ldots, T}$ are positive semidefinite, as one can easily show inductively from the fact that

$$
\begin{aligned}
L_{t+1} & =\left[\begin{array}{cc}
Q+A^{\mathrm{T}} X_{t+1} A & S+A^{\mathrm{T}} X_{t+1} B \\
S^{\mathrm{T}}+B^{\mathrm{T}} X_{t+1} A & R+B^{\mathrm{T}} X_{t+1} B
\end{array}\right] \\
& =\Pi+\left[\begin{array}{c}
A^{\mathrm{T}} \\
B^{\mathrm{T}}
\end{array}\right] X_{t+1}\left[\begin{array}{ll}
A & B
\end{array}\right] \geq 0 .
\end{aligned}
$$

In fact, $X_{t}$ is the generalised Schur complement of the block submatrix in the top left of $L_{t+1}$, and is therefore positive semidefinite. Another consequence is the fact that the following subspace inclusion holds for every $t \in\{0, \ldots, T-1\}$ :

$$
\operatorname{ker}\left(R+B^{\mathrm{T}} X_{t+1} B\right) \subseteq \operatorname{ker}\left(A^{\mathrm{T}} X_{t+1} B+S\right) .
$$

As aforementioned, the solution of $\operatorname{GRDP}(\Sigma, P)$ provides the optimal feedback matrix of the classic finite-horizon LQ problem under the weakest assumptions on the Popov triple $\Sigma=(A, B, \Pi)$. More precisely, consider the discrete linear time-invariant system governed by the difference equation

$$
x_{t+1}=A x_{t}+B u_{t},
$$

where $A \in \mathbb{R}^{n \times n}$ and $B \in \mathbb{R}^{n \times m}$, and where, for all $t \geq 0$, $x_{t} \in \mathbb{R}^{n}$ represents the state and $u_{t} \in \mathbb{R}^{m}$ represents the control input. Let the initial state $x_{0} \in \mathbb{R}^{n}$ be given. The problem is to find a sequence of inputs $u_{t}$, with $t=0,1, \ldots, T-1$, minimising the cost function

$J\left(x_{0}, u\right) \triangleq \sum_{t=0}^{T-1}\left[\begin{array}{ll}x_{t}^{\mathrm{T}} & u_{t}^{\mathrm{T}}\end{array}\right]\left[\begin{array}{cc}Q & S \\ S^{\mathrm{T}} & R\end{array}\right]\left[\begin{array}{l}x_{t} \\ u_{t}\end{array}\right]+x_{T}^{\mathrm{T}} P x_{T}$. 
For any time $t$, the set $\mathscr{U}_{t}$ of all optimal inputs can be written as $\mathscr{U}_{t}=\left\{-K_{t} x_{t}+G_{t} v_{t}\right\}$, where

$$
\begin{aligned}
& K_{t}=\left(R+B^{\mathrm{T}} X_{t+1} B\right)^{\dagger}\left(S^{\mathrm{T}}+B^{\mathrm{T}} X_{t+1} A\right), \\
& G_{t}=I_{m}-\left(R+B^{\mathrm{T}} X_{t+1} B\right)^{\dagger}\left(R+B^{\mathrm{T}} X_{t+1} B\right),
\end{aligned}
$$

in which $X_{t}$ is the solution of $\operatorname{GRDP}(\Sigma, P)$. The term $G_{t} v_{t}$ in the set $\mathscr{U}_{t}$ of all optimal controls in $t \in\{0, \ldots, T-1\}$ does not influence the cost, which means that $v_{t} \in \mathbb{R}^{m}$ is completely arbitrary. The equation characterising the set of optimal state trajectories is

$$
x_{t+1}=\left(A-B K_{t}\right) x_{t}-B G_{t} v_{t},
$$

where as aforementioned $v_{t} \in \mathbb{R}^{m}$ is arbitrary. The optimal cost is $J^{*}=x_{0}^{\mathrm{T}} X_{0} x_{0}$.

\section{The Generalised Discrete Algebraic Riccati EQUATION}

We begin this section by recalling a standard linear algebra result that is used in the derivations throughout the paper.

Lemma 2.1: Consider $P=\left[\begin{array}{ll}P_{11} & P_{12} \\ P_{12}^{\mathrm{T}} & P_{22}\end{array}\right]=P^{\mathrm{T}} \geq 0$. Then,

(i) $\operatorname{ker} P_{12} \supseteq \operatorname{ker} P_{22}$;

(ii) $P_{12} P_{22}^{\dagger} P_{22}=P_{12}$;

(iii) $P_{12}\left(I-P_{22}^{\dagger} P_{22}\right)=0$;

(iv) $P_{11}-P_{12} P_{22}^{\dagger} P_{12}^{\mathrm{T}} \geq 0$.

We now introduce the so-called Generalised Discrete Algebraic Riccati Equation $\operatorname{GDARE}(\Sigma)$, defined as

$X=A^{\mathrm{T}} X A-\left(A^{\mathrm{T}} X B+S\right)\left(R+B^{\mathrm{T}} X B\right)^{\dagger}\left(B^{\mathrm{T}} X A+S^{\mathrm{T}}\right)+Q$.

The algebraic equation $\operatorname{GDARE}(\Sigma)$ subject to the constraint

$$
\operatorname{ker}\left(R+B^{\mathrm{T}} X B\right) \subseteq \operatorname{ker}\left(A^{\mathrm{T}} X B+S\right)
$$

is usually referred to as Constrained Generalised Discrete Algebraic Riccati Equation $\operatorname{CGDARE}(\Sigma)$. Observe that $\operatorname{CGDARE}(\Sigma)$ constitutes a generalisation of the classic Discrete Riccati Algebraic Equation $\operatorname{DARE}(\Sigma)$

$X=A^{\mathrm{T}} X A-\left(A^{\mathrm{T}} X B+S\right)\left(R+B^{\mathrm{T}} X B\right)^{-1}\left(B^{\mathrm{T}} X A+S^{\mathrm{T}}\right)+Q$,

in the sense that any solution of $\operatorname{DARE}(\Sigma)$ is also a solution of $\operatorname{CGDARE}(\Sigma)$ but the vice-versa is not true in general. Importantly, however, the inertia of $R+B^{\mathrm{T}} X B$ is independent of the particular solution of the $\operatorname{CGDARE}(\Sigma)$, [11, Theorem 2.4]. This implies that a given CGDARE $(\Sigma)$ cannot have one solution $X=X^{\mathrm{T}}$ such that $R+B^{\mathrm{T}} X B$ is non-singular and another solution $Y=Y^{\mathrm{T}}$ for which $R+B^{\mathrm{T}} Y B$ is singular. As such, i) if $X$ is a solution of $\operatorname{DARE}(\Sigma)$, then all solutions of $\operatorname{CGDARE}(\Sigma)$ will also satisfy $\operatorname{DARE}(\Sigma)$ and, ii) if $X$ is a solution of $\operatorname{CGDARE}(\Sigma)$ such that $R+B^{\mathrm{T}} X B$ is singular, then $\operatorname{DARE}(\Sigma)$ does not admit solutions.

To any solution $X=X^{\mathrm{T}} \in \mathbb{R}^{n \times n}$ of $\operatorname{GDARE}(\Sigma)$ we can associate the corresponding gain matrix $K_{X}$ defined as

$$
K_{X} \triangleq\left(R+B^{\mathrm{T}} X B\right)^{\dagger}\left(B^{\mathrm{T}} X A+S^{\mathrm{T}}\right)
$$

and the associated closed-loop matrix $A_{X} \triangleq A-B K_{X}$.

Notice that all symmetric positive semidefinite solutions of $\operatorname{GDARE}(\Sigma)$ satisfy (8), and are therefore solutions of $\operatorname{CGDARE}(\Sigma)$. In fact, if $X$ is positive semidefinite, we find

$$
\left[\begin{array}{cc}
A^{\mathrm{T}} X A+Q & A^{\mathrm{T}} X B+S \\
B^{\mathrm{T}} X A+S^{\mathrm{T}} & R+B^{\mathrm{T}} X B
\end{array}\right]=\left[\begin{array}{c}
A^{\mathrm{T}} \\
B^{\mathrm{T}}
\end{array}\right] X\left[\begin{array}{ll}
A & B
\end{array}\right]+\Pi \geq 0 .
$$

Therefore, applying (i) of Lemma 2.1 we find (8).

For the sake of simplicity, from now on, given a symmetric matrix $X=X^{\mathrm{T}}$, we define

$$
\begin{aligned}
& R_{X} \triangleq R+B^{\mathrm{T}} X B \\
& S_{X} \triangleq A^{\mathrm{T}} X B+S
\end{aligned}
$$

so that (8) can be written as $\operatorname{ker} R_{X} \subseteq \operatorname{ker} S_{X}$. We also define $G_{X} \triangleq I_{m}-R_{X}^{\dagger} R_{X}$. Hence, if (8) holds, we have $S_{X} G_{X}=0$. Notice also that

$$
\operatorname{ker} R_{X}=\operatorname{im} G_{X}
$$

In fact, it is trivial to see that $\operatorname{ker} R_{X} \supseteq \operatorname{im} G_{X}$, since $R_{X}\left(I_{m}-\right.$ $\left.R_{X}^{\dagger} R_{X}\right)=R_{X}-R_{X} R_{X}^{\dagger} R_{X}=0$. It is also easy to see that the opposite inclusion holds. Indeed, if $\xi \in \operatorname{ker} R_{X}$, then $\left(I_{m}-R_{X}^{\dagger} R_{X}\right) \xi=\xi$, so that $\xi \in \operatorname{im} G_{X}$. The term $R_{X}^{\dagger} R_{X}$ is the orthogonal projector that projects onto $\operatorname{im} R_{X}^{\dagger}=\operatorname{im} R_{X}$ so that $G_{X}$ is the orthogonal projector that projects onto $\operatorname{ker} R_{X}$.

\section{GDARE AND THE EXTENDED SYMPLECTIC PENCIL}

In this section we adapt the analysis carried out in [7] for standard discrete algebraic Riccati equations to the case of CGDARE $(\Sigma)$. Consider the so-called extended symplectic pencil $N-z M$, where

$$
M \triangleq\left[\begin{array}{ccc}
I_{n} & O & O \\
O & -A^{\mathrm{T}} & O \\
O & -B^{\mathrm{T}} & O
\end{array}\right], \quad N \triangleq\left[\begin{array}{ccc}
A & O & B \\
Q & -I_{n} & S \\
S^{\mathrm{T}} & O & R
\end{array}\right] .
$$

Here we do not assume that the matrix pencil $N-z M$ is regular. We begin by giving a necessary and sufficient condition for $N$ to be singular. We will also show that, unlike the case in which the pencil $N-z M$ is regular, this is not equivalent to the fact that $N-z M$ has a generalised eigenvalue at zero.

Lemma 3.1: Matrix $N$ is singular if and only if $\left[\begin{array}{cc}A & B \\ S^{\mathrm{T}} & R\end{array}\right]$ is singular.

Proof: First, we can see that $N$ is singular if and only if $\left[\begin{array}{cc}A & B \\ S^{\mathrm{T}} & R\end{array}\right]$ is singular, since $\left[\begin{array}{lll}v_{1}{ }^{\mathrm{T}} & v_{2}{ }^{\mathrm{T}} & v_{3}{ }^{\mathrm{T}}\end{array}\right] N=0$ if and only if $v_{2}=0$ and $\left[\begin{array}{ll}v_{1}{ }^{\mathrm{T}} & v_{3}{ }^{\mathrm{T}}\end{array}\right]\left[\begin{array}{cc}A & B \\ S^{\mathrm{T}} & R\end{array}\right]=0$.

Lemma 3.2: Matrix $N$ is singular if and only if at least one of the two matrices $R$ and $A-B R^{\dagger} S^{\mathrm{T}}$ is singular.

Proof: (If). When $R$ is singular, a non-zero vector $v_{3}$ exists such $v_{3}{ }^{\mathrm{T}} R=0$. Since $\operatorname{ker} R \subseteq \operatorname{ker} S$, then we have also $\left[\begin{array}{ll}0 & v_{3}^{\mathrm{T}}\end{array}\right]\left[\begin{array}{cc}A & B \\ S^{\mathrm{T}} & R\end{array}\right]=0$. If $R$ is invertible but $A-$ $B R^{\dagger} S^{\mathrm{T}}=A-B R^{-1} S^{\mathrm{T}}$ is singular, there exists a non-zero vector $v$ such that $v^{\mathrm{T}}\left(A-B R^{-1} S^{\mathrm{T}}\right)=0$. This means that $\left[\begin{array}{ll}v^{\mathrm{T}} & -v^{\mathrm{T}} B R^{-1}\end{array}\right]\left[\begin{array}{cc}A & B \\ S^{\mathrm{T}} & R\end{array}\right]=0$. In both cases, by Lemma 
$3.1, N$ is singular.

(Only if). When both $R$ and $A-B R^{-1} S^{\mathrm{T}}$ are non-singular, a direct check shows that

$$
\left[\begin{array}{cc}
A & B \\
S^{\mathrm{T}} & R
\end{array}\right]=\left[\begin{array}{cc}
A-B R^{-1} S^{\mathrm{T}} & B \\
O & R
\end{array}\right]\left[\begin{array}{cc}
I_{n} & O \\
R^{-1} S^{\mathrm{T}} & I_{m}
\end{array}\right] .
$$

Since both matrices in the right-hand side are non-singular, the matrix in the left-hand side is invertible, and such is $N$.

In the following theorem we present a useful decomposition of the extended symplectic pencil that parallels the classic one - see e.g. [7] - which is valid in the case in which the pencil $N-z M$ is regular.

Theorem 3.1: Let $X$ be a symmetric solution of $\operatorname{CGDARE}(\Sigma)$. Let also $K_{X}$ be the associated gain and $A_{X}$ be the associated closed-loop matrix. Two invertible matrices $U_{X}$ and $V_{X}$ exist such that

$$
U_{X}(N-z M) V_{X}=\left[\begin{array}{ccc}
A_{X}-z I_{n} & O & B \\
O & I_{n}-z A_{X}^{\mathrm{T}} & O \\
O & -z B^{\mathrm{T}} & R_{X}
\end{array}\right] .
$$

Proof: The statement follows by considering the invertible matrices

$U_{X} \triangleq\left[\begin{array}{ccc}I_{n} & O & O \\ A_{X}^{\mathrm{T}} X & I_{n} & -K_{X}^{\mathrm{T}} \\ B^{\mathrm{T}} X & O & I_{m}\end{array}\right] \quad$ and $\quad V_{X} \triangleq\left[\begin{array}{ccc}I_{n} & O & O \\ X & -I_{n} & O \\ -K_{X} & O & I_{m}\end{array}\right]$

From Theorem 3.1 we find that if $X$ is a solution of $\operatorname{CGDARE}(\Sigma)$, in view of the triangular structure obtained above we have

$$
\operatorname{det}(N-z M)=\operatorname{det}\left(A_{X}-z I_{n}\right) \cdot \operatorname{det}\left(I_{n}-z A_{X}^{\mathrm{T}}\right) \cdot \operatorname{det} R_{X} .
$$

When $R_{X}$ is non-singular, the dynamics represented by this matrix pencil are decomposed into a part governed by the generalised eigenstructure of $A_{X}-z I_{n}$, a part governed by the finite generalised eigenstructure of $I_{n}-z A_{X}^{\mathrm{T}}$, and a part which corresponds to the dynamics of the eigenvalues at infinity. Then, in particular when $R_{X}$ is non-singular the singular part of $A_{X}$ does not depend on the solution of $\operatorname{CGDARE}(\Sigma)$. When $X$ is a solution of $\operatorname{DARE}(\Sigma)$, the generalised eigenvalues ${ }^{2}$ of $N-z M$ are given by the eigenvalues of $A_{X}$, the reciprocal of the non-zero eigenvalues of $A_{X}$, and a generalised eigenvalues at infinity whose algebraic multiplicity is equal to $m$ plus the algebraic multiplicity of the eigenvalue of $A_{X}$ at the origin. The matrix pencil $I_{n}-z A_{X}^{\mathrm{T}}$ has no generalised eigenvalues at $z=0$. This means that $z=0$ is a generalised eigenvalue of the matrix pencil $U_{X}(N-z M) V_{X}$ if and only if it is a generalised eigenvalue of the matrix pencil $A_{X}-z I_{n}$, because certainly $z=0$ cannot cause the rank of $I_{n}-z A_{X}^{\mathrm{T}}$ to be smaller than its normal rank and because the normal rank of $N-z M$ is $2 n+m$. This means that the Kronecker eigenstructure of the eigenvalue at the origin of $U_{X}(N-z M) V_{X}$ coincides with the Jordan eigenstructure of

\footnotetext{
${ }^{2}$ Recall that a generalised eigenvalue of a matrix pencil $N-z M$ is a value of $z \in \mathbb{C}$ for which the rank of the matrix pencil $N-z M$ is lower than its normal rank.
}

the eigenvalue at the origin of the closed-loop matrix $A_{X}$. Since the generalised eigenvalues of $N-z M$ do not depend on the particular solution $X=X^{\mathrm{T}}$ of $\operatorname{CGDARE}(\Sigma)$, the same holds for the generalised eigenvalues and the Kronecker structure of $U_{X}(N-z M) V_{X}$ for any non-singular $U_{X}$ and $V_{X}$. Therefore, the nilpotent structure of the closed-loop matrix $A_{X}$ - which is the Jordan eigenstructure of the generalised eigenvalue at the origin of $A_{X}$ - if any, is independent of the particular solution $X=X^{\mathrm{T}}$ of $\operatorname{CGDARE}(\Sigma)$. Moreover, since

$$
U_{X} N V_{X}=\left[\begin{array}{ccc}
A_{X} & O & B \\
O & I_{n} & O \\
O & O & R_{X}
\end{array}\right]
$$

we see that when $R_{X}$ is invertible $N$ is singular if and only if $A_{X}$ is singular. Therefore, from the observations in the beginning of this section, we also have the following result.

Lemma 3.3: (see e.g. [2]) Let $R_{X}$ be invertible. Then, $A_{X}$ is singular if and only if at least one of the two matrices $R$ and $A-B R^{\dagger} S^{\mathrm{T}}$ is singular.

However, when the matrix $R_{X}$ is singular, it is no longer true that $A_{X}$ is singular if and only if $R$ or $A-B R^{\dagger} S^{\mathrm{T}}$ is singular. Indeed, (13) shows that the algebraic multiplicity of the eigenvalue at the origin of $N$ is equal to the sum of the algebraic multiplicities of the eigenvalue at the origin of $A_{X}$ and $R_{X}$. Therefore, the fact that $N$ is singular does not necessarily imply that $A_{X}$ is singular.

Proposition 3.1: The closed-loop matrix $A_{X}$ is singular if and only if $\operatorname{rank} R<\operatorname{rank} R_{X}$ or $A-B R^{\dagger} S^{\mathrm{T}}$ is singular.

Proof: Given a square matrix $Z$, let us denote by $\mu(Z)$ the algebraic multiplicity of its eigenvalue at the origin. Then, we know from (13) that $\mu(N)=\mu\left(\left[\begin{array}{cc}A & B \\ S^{\mathrm{T}} & R\end{array}\right]\right)=\mu\left(A_{X}\right)+\mu\left(R_{X}\right)$. Consider a basis in the input space that isolates the invertible part of $R$. In other words, in this basis $R$ is written as $R=$ $\left[\begin{array}{ll}R_{1} & O \\ O & O\end{array}\right]$ where $R_{1}$ is invertible, while $B=\left[\begin{array}{cc}B_{1} & B_{2}\end{array}\right]$ and $S=\left[\begin{array}{ll}S_{1} & O\end{array}\right]$ are partitioned accordingly. It follows that $\mu\left(\left[\begin{array}{cc}A & B \\ S^{\mathrm{T}} & R\end{array}\right]\right)=\mu(R)+\mu\left(\left[\begin{array}{cc}A & B_{1} \\ S_{1}^{\mathrm{T}} & R_{1}\end{array}\right]\right)$. As such,

$$
\begin{aligned}
\mu\left(A_{X}\right) & =\mu\left(\left[\begin{array}{cc}
A & B \\
S^{\mathrm{T}} & R
\end{array}\right]\right)-\mu\left(R_{X}\right) \\
& =\mu\left(\left[\begin{array}{cc}
A & B_{1} \\
S_{1}^{\mathrm{T}} & R_{1}
\end{array}\right]\right)+\mu(R)-\mu\left(R_{X}\right) .
\end{aligned}
$$

First, we show that if $\operatorname{rank} R<\operatorname{rank} R_{X}$, then $A_{X}$ is singular. Since $\operatorname{rank} R<\operatorname{rank} R_{X}$, then obviously $\mu(R)>\mu\left(R_{X}\right)$, so that (14) gives $\mu\left(A_{X}\right)>0$.

Let now $A-B R^{\dagger} S^{\mathrm{T}}$ be singular, and let $\operatorname{rank} R=\operatorname{rank} R_{X}$. From (14) we find that $\mu\left(A_{X}\right)=\mu\left(\left[\begin{array}{cc}A & B_{1} \\ S_{1}^{\mathrm{T}} & R_{1}\end{array}\right]\right)$. However, $A-$ $B R^{\dagger} S^{\mathrm{T}}=A-B_{1} R_{1}^{-1} S_{1}^{\mathrm{T}}$. If $A-B R^{\dagger} S^{\mathrm{T}}$ is singular, there exists a non-zero vector $k$ such that $\left[\begin{array}{lll}k^{\mathrm{T}} & -k^{\mathrm{T}} B_{1} R_{1}^{-1}\end{array}\right]\left[\begin{array}{cc}A & B_{1} \\ S_{1}^{\mathrm{T}} & R_{1}\end{array}\right]=$ 0. Hence, $\mu\left(\left[\begin{array}{cc}A & B_{1} \\ S_{1}^{\mathrm{T}} & R_{1}\end{array}\right]\right)>0$, and therefore also $\mu\left(A_{X}\right)>0$. The converse can be proved with a similar argument, using again (14).

Remark 3.1: We recall that the algebraic multiplicity of the eigenvalue at the origin of $R_{X}$ is invariant for any solution 
$X$ of $\operatorname{CGDARE}(\Sigma),[11]$. Hence, as a direct consequence of (14), we have that the algebraic multiplicity of the eigenvalue at the origin of $A_{X}$ is the same for any solution $X$ of $\operatorname{CGDARE}(\Sigma)$. This means, in particular, that the closed-loop matrix corresponding to a given a solution of $\operatorname{CGDARE}(\Sigma)$ is singular if and only if the closed-loop matrix corresponding to any other solution of $\operatorname{CGDARE}(\Sigma)$ is singular. In the next section we show that a stronger result holds: when present, the zero eigenvalue has the same Jordan structure for any pair $A_{X}$ and $A_{Y}$ of closed-loop matrices corresponding to any pair $X, Y$ of solutions of CGDARE $(\Sigma)$. Moreover, the generalised eigenspaces corresponding to the zero eigenvalue of $A_{X}$ and $A_{Y}$ coincide. The restriction of $A_{X}$ and $A_{Y}$ to this generalised eigenspace also coincide. Finally, $X$ and $Y$ coincide along this generalised eigenspace.

\section{The SUBSPACE $\mathscr{U}$}

Given a solution $X=X^{\mathrm{T}}$ of $\operatorname{CGDARE}(\Sigma)$, we denote by $\mathscr{U}$ the generalised eigenspace corresponding to the eigenvalue at the origin of $A_{X}$, [7]. In formulae, $\mathscr{U} \triangleq \operatorname{ker} A_{X}^{n}$. In this section we want to prove that all solutions of $\operatorname{CGDARE}(\Sigma)$ are coincident along $\mathscr{U}$. In other words, given two solutions $X=X^{\mathrm{T}}$ and $Y=Y^{\mathrm{T}}$ of $\operatorname{GDARE}(\Sigma)$, we show that $\mathscr{U} \subseteq$ $\operatorname{ker}(X-Y)$. Stated differently, given a basis matrix ${ }^{3} U$ of the subspace $\mathscr{U}$, the change of coordinate matrix $T=\left[\begin{array}{ll}U & U_{c}\end{array}\right]$ yields

$T^{-1} X T=\left[\begin{array}{ll}X_{11} & X_{12} \\ X_{12}^{\mathrm{T}} & X_{22}\end{array}\right]$ and $T^{-1} Y T=\left[\begin{array}{ll}X_{11} & X_{12} \\ X_{12}^{\mathrm{T}} & Y_{22}\end{array}\right]$.

We first present two results that will be useful to prove this point. Let $X=X^{\mathrm{T}} \in \mathbb{R}^{n \times n}$. Similarly to [7], we define the function

$$
\begin{gathered}
\mathscr{D}(X) \triangleq X-A^{\mathrm{T}} X A+\left(A^{\mathrm{T}} X B+S\right)\left(R+B^{\mathrm{T}} X B\right)^{\dagger} \\
\cdot\left(B^{\mathrm{T}} X A+S^{\mathrm{T}}\right)-Q .
\end{gathered}
$$

If in particular $X=X^{\mathrm{T}}$ is a solution of $\operatorname{GDARE}(\Sigma)$, then $\mathscr{D}(X)=0$. For the sake of conciseness, we recall that given two solutions $X=X^{\mathrm{T}} \in \mathbb{R}^{n \times n}$ and $Y=Y^{\mathrm{T}} \in \mathbb{R}^{n \times n}$ of $\operatorname{GDARE}(\Sigma)$, we have defined $R_{X}=R+B^{\mathrm{T}} X B, S_{X}=$ $A^{\mathrm{T}} X B+S$ and $R_{Y}=R+B^{\mathrm{T}} Y B, S_{Y} \triangleq A^{\mathrm{T}} Y B+S$.

Lemma 4.1: Let $X=X^{\mathrm{T}} \in \mathbb{R}^{n \times n}$ and $Y=Y^{\mathrm{T}} \in \mathbb{R}^{n \times n}$ be such that (8) holds, i.e.,

$$
\begin{aligned}
& \operatorname{ker} R_{X} \subseteq \operatorname{ker} S_{X} \\
& \operatorname{ker} R_{Y} \subseteq \operatorname{ker} S_{Y} .
\end{aligned}
$$

Let $A_{X}=A-B K_{X}$ with $K_{X}=R_{X}^{\dagger} S_{X}^{\mathrm{T}}$ and $A_{Y}=A-B K_{Y}$ with $K_{Y}=R_{Y}^{\dagger} S_{Y}^{\mathrm{T}}$. Moreover, let us define the difference $\Delta \triangleq X-Y$. Then,

$$
\mathscr{D}(X)-\mathscr{D}(Y)=\Delta-A_{Y}^{\mathrm{T}} \Delta A_{Y}+A_{Y}^{\mathrm{T}} \Delta B R_{X}^{\dagger} B^{\mathrm{T}} \Delta A_{Y} .
$$

The proof can be found in [1, p.382].

The following lemma is a generalisation of Lemma 2.2 in [7] to the case of a closed-loop matrix in which the term $\left(R+B^{\mathrm{T}} X B\right)$ appears with the pseudo-inverse instead of the inverse.

\footnotetext{
${ }^{3}$ Given a subspace $\mathscr{S}$, a basis matrix of $\mathscr{S}$ is a matrix $S$ such that $\operatorname{im} S=\mathscr{S}$ and $\operatorname{ker} S=\{0\}$.
}

Lemma 4.2: Let $X=X^{\mathrm{T}} \in \mathbb{R}^{n \times n}$ and $Y=Y^{\mathrm{T}} \in \mathbb{R}^{n \times n}$ be such that (17-18) hold. Then,

$$
\mathscr{D}(X)-\mathscr{D}(Y)=\Delta-A_{Y}^{\mathrm{T}} \Delta A_{X} .
$$

The following result is an extension of Proposition 2.1 in [7] to solutions of $\operatorname{CGDARE}(\Sigma)$.

Theorem 4.1: All solutions of $\operatorname{CGDARE}(\Sigma)$ are coincident along $\mathscr{U}$, i.e., given two solutions $X$ and $Y$ of $\operatorname{CGDARE}(\Sigma)$,

$$
(X-Y) \mathscr{U}=\{0\} ;
$$

Moreover, $\mathscr{U}$ does not depend on the solution $X$ of $\operatorname{CGDARE}(\Sigma)$, i.e., given two solutions $X$ and $Y$ of $\operatorname{CGDARE}(\Sigma)$, there holds

$$
\operatorname{ker} A_{X}^{n}=\operatorname{ker} A_{Y}^{n} \text {. }
$$

The proof is omitted for the sake of brevity. The proof of this result follows the same lines of that of [7, Proposition 2.1], and uses the generalised result given in Lemma 4.2.

\section{The Generalised Riccati Difference Equation}

Consider the $\operatorname{GRDE}(\Sigma)$ along with the terminal condition $X_{T}=P=P^{\mathrm{T}} \geq 0$. Let us define

$\mathscr{R}(X) \triangleq A^{\mathrm{T}} X A-\left(A^{\mathrm{T}} X B+S\right)\left(R+B^{\mathrm{T}} X B\right)^{\dagger}\left(B^{\mathrm{T}} X A+S^{\mathrm{T}}\right)+Q$.

With this definition, $\operatorname{GRDE}(\Sigma)$ can be written as $X_{t}=$ $\mathscr{R}\left(X_{t+1}\right)$. Moreover, GDARE $(\Sigma)$ can be written as

$$
\mathscr{D}(X)=X-\mathscr{R}(X)=0 .
$$

With a slight abuse of nomenclature, we use the term nilpotency index of a matrix $M$ to refer to the smallest integer $v$ for which $\operatorname{ker} M^{v}=\operatorname{ker} M^{v+1}$, which is defined also when $M$ is not nilpotent. We have the following important result.

Theorem 5.1: Let $X_{0}=X_{0}^{\mathrm{T}}$ be a solution of $\operatorname{CGDARE}(\Sigma)$. Let $v$ be the index of nilpotency of $A_{X_{0}}$. Moreover, let $X_{t}$ be a solution of (1-2) and define $\Delta_{t} \triangleq X_{t}-X_{0}$. Then, for $\tau \geq v$, we have $\Delta_{T-\tau} \mathscr{U}=\{0\}$.

Proof: Since $X_{0}=X_{0}^{\mathrm{T}}$ is a solution of $\operatorname{CGDARE}(\Sigma)$, we have $\mathscr{D}\left(X_{0}\right)=0$. This is equivalent to saying that $X_{0}=\mathscr{R}\left(X_{0}\right)$. From the definition $\Delta_{t} \triangleq X_{t}-X_{0}$ we get in particular $\Delta_{T}=$ $X_{T}-X_{0}$. With these definitions in mind, we find

$$
\begin{aligned}
\Delta_{t} & =\mathscr{R}\left(X_{t+1}\right)-\mathscr{R}\left(X_{0}\right)=X_{t+1}-\mathscr{D}\left(X_{t+1}\right)-X_{0} \\
& =\Delta_{t+1}-\mathscr{D}\left(X_{t+1}\right)=\Delta_{t+1}-\mathscr{D}\left(X_{t+1}\right)+\mathscr{D}\left(X_{0}\right) \\
& =\Delta_{t+1}-\left[\mathscr{D}\left(X_{t+1}\right)-\mathscr{D}\left(X_{0}\right)\right] .
\end{aligned}
$$

However, we know from (19) that

$$
\begin{aligned}
\mathscr{D}\left(X_{t+1}\right)-\mathscr{D}\left(X_{0}\right)=\Delta_{t+1}-A_{X_{0}}^{\mathrm{T}}\left[\Delta_{t+1}\right. \\
\left.\quad-\Delta_{t+1} B\left(R+B^{\mathrm{T}} X_{t+1} B\right)^{\dagger} B^{\mathrm{T}} \Delta_{t+1}\right] A_{X_{0}}(22)
\end{aligned}
$$

which, once plugged into (21), gives

$$
\begin{aligned}
\Delta_{t}= & \Delta_{t+1}-\Delta_{t+1}+A_{X_{0}}^{\mathrm{T}}\left[\Delta_{t+1}\right. \\
& \left.+\Delta_{t+1} B\left(R+B^{\mathrm{T}} X_{t+1} B\right)^{\dagger} B^{\mathrm{T}} \Delta_{t+1}\right] A_{X_{0}} \\
= & A_{X_{0}}^{\mathrm{T}}\left[I_{n}-\Delta_{t+1} B\left(R+B^{\mathrm{T}} X_{t+1} B\right)^{\dagger} B^{\mathrm{T}}\right] \Delta_{t+1} A_{X_{0}} \\
= & F_{t+1} \Delta_{t+1} A_{X_{0}},
\end{aligned}
$$


where

$$
F_{t+1} \triangleq A_{X_{0}}^{\mathrm{T}}-A_{X_{0}}^{\mathrm{T}} \Delta_{t+1} B\left(R+B^{\mathrm{T}} X_{t+1} B\right)^{\dagger} B^{\mathrm{T}}
$$

It follows that we can write

$$
\begin{aligned}
\Delta_{T-1} & =F_{T} \Delta_{T} A_{X_{0}}, \\
\Delta_{T-2} & =F_{T-1} \Delta_{T-1} A_{X_{0}}=F_{T-1} F_{T} \Delta_{T} A_{X_{0}}^{2}, \\
& \vdots \\
\Delta_{T-\tau} & =\left(\prod_{i=T-\tau+1}^{T} F_{i}\right) \Delta_{T} A_{X_{0}}^{\tau} .
\end{aligned}
$$

This shows that for $\tau \geq v$ we have $\operatorname{ker} \Delta_{T-\tau} \supseteq \operatorname{ker} A_{X_{0}}^{n}$.

Now we show that the result given in Theorem 5.1 can be used to obtain a reduction for the generalised discretetime Riccati difference equation. Consider the same basis induced by the change of coordinates used in Theorem 4.1, so that the first $v$ components of this basis span the subspace $\mathscr{U}=\operatorname{ker} A_{X}^{n}$. The closed-loop matrix in this basis can be written as

$$
A_{X_{0}}=\left[\begin{array}{cc}
N_{0} & \star \\
O & Z
\end{array}\right]
$$

where $N_{0}$ is nilpotent and $Z$ is non-singular. Hence, $A_{X_{0}}^{v}=$ $\left[\begin{array}{cc}O & \star \\ O & Z^{v}\end{array}\right]$, where we recall that $v$ is the nilpotency index of $A_{X}$. By writing (25) in this basis, for $\tau \geq v$ we find

$\Delta_{T-\tau}=\left[\begin{array}{cc}\star & \star \\ \star & \star\end{array}\right]\left[\begin{array}{cc}O & \star \\ O & Z^{v}\end{array}\right]=\left[\begin{array}{cc}O & \star \\ O & \star\end{array}\right]=\left[\begin{array}{ll}O & O \\ O & \star\end{array}\right]$,

where the last equality follows from the fact that $\Delta_{T-\tau}$ is symmetric.

Now, let us rewrite the Riccati difference equation (23) as

$$
\Delta_{t}=A_{X_{0}}^{\mathrm{T}} \Delta_{t+1} A_{X_{0}}-A_{X_{0}}^{\mathrm{T}} \Delta_{t+1} B\left(R+B^{\mathrm{T}} X_{t+1} B\right)^{\dagger} B^{\mathrm{T}} \Delta_{t+1} A_{X_{0}}
$$

For $t \leq T-v$, we get $\Delta=\left[\begin{array}{cc}O & O \\ O & \Psi_{t}\end{array}\right]$, and the previous equation becomes

$$
\begin{aligned}
& {\left[\begin{array}{ll}
O & O \\
O & \Psi_{t}
\end{array}\right]=\left[\begin{array}{cc}
N_{0}^{\mathrm{T}} & O \\
\star & Z^{\mathrm{T}}
\end{array}\right]\left[\begin{array}{cc}
O & O \\
O & \Psi_{t+1}
\end{array}\right]\left[\begin{array}{cc}
N_{0} & \star \\
O & Z
\end{array}\right]} \\
& -\left[\begin{array}{cc}
N_{0}^{\mathrm{T}} & O \\
\star & Z^{\mathrm{T}}
\end{array}\right]\left[\begin{array}{cc}
O & O \\
O & \Psi_{t+1}
\end{array}\right] B\left(R+B^{\mathrm{T}} X_{t+1} B\right)^{\dagger} B^{\mathrm{T}}\left[\begin{array}{cc}
O & O \\
O & \Psi_{t+1}
\end{array}\right]\left[\begin{array}{cc}
N_{0} & \star \\
O & Z
\end{array}\right] \\
& =\left[\begin{array}{lc}
O & O \\
O & Z^{\mathrm{T}} \Psi_{t+1} Z
\end{array}\right] \\
& -\left[\begin{array}{cc}
O & O \\
O & Z^{\mathrm{T}} \Psi_{t+1}
\end{array}\right]\left[\begin{array}{l}
B_{1} \\
B_{2}
\end{array}\right]\left(R+\left[\begin{array}{cc}
B_{1}^{\mathrm{T}} & B_{2}^{\mathrm{T}}
\end{array}\right]\left(\Delta_{t+1}+X_{0}\right)\left[\begin{array}{l}
B_{1} \\
B_{2}
\end{array}\right]\right)^{\dagger} \\
& \cdot\left[\begin{array}{ll}
B_{1}^{\mathrm{T}} & B_{2}^{\mathrm{T}}
\end{array}\right]\left[\begin{array}{lc}
O & O \\
O & \Psi_{t+1} Z
\end{array}\right] \text {. }
\end{aligned}
$$

By partitioning $X_{0}$ as $X_{0}=\left[\begin{array}{ll}X_{0,11} & X_{0,12} \\ X_{0,12}^{\mathrm{T}} & X_{0,22}\end{array}\right]$, we get

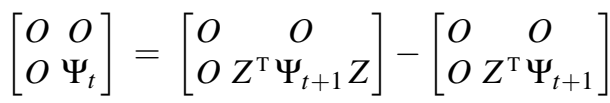

$$
\begin{aligned}
& \cdot\left[\begin{array}{lc}
\star & \star \\
\star B_{2}\left(R_{0}+B_{2}^{\mathrm{T}} \Psi_{t+1} B_{2}\right)^{\dagger} B_{2}^{\mathrm{T}}
\end{array}\right]\left[\begin{array}{ll}
O & O \\
O & \Psi_{t+1} Z
\end{array}\right]
\end{aligned}
$$

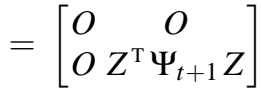

$$
\begin{aligned}
& -\left[\begin{array}{lc}
O & O \\
O & Z^{\mathrm{T}} \Psi_{t+1} B_{2}\left(R_{0}+B_{2}^{\mathrm{T}} \Psi_{t+1} B_{2}\right)^{\dagger} B_{2}^{\mathrm{T}} \Psi_{t+1} Z
\end{array}\right],
\end{aligned}
$$

where $R_{0} \triangleq R+B_{2}^{\mathrm{T}} X_{0,22} B_{2}$. Therefore, $\Psi_{t}$ satisfies the reduced homogeneous Riccati difference equation

$\Psi_{t}=Z^{\mathrm{T}} \Psi_{t+1} Z-Z^{\mathrm{T}} \Psi_{t+1} B_{2}\left(R_{0}+B_{2}^{\mathrm{T}} \Psi_{t+1} B_{2}\right)^{\dagger} B_{2}^{\mathrm{T}} \Psi_{t+1} Z$.

The associated generalised discrete Riccati algebraic equation is

$$
\Psi-Z^{\mathrm{T}} \Psi Z+Z^{\mathrm{T}} \Psi B_{2}\left(R_{0}+B_{2}^{\mathrm{T}} \Psi B_{2}\right)^{\dagger} B_{2}^{\mathrm{T}} \Psi Z=0 .
$$

Being homogeneous, this equation admits the solution $\Psi=0$. This fact has two important consequences:

- The closed-loop matrix associated with this solution is clearly $Z$, which is non-singular. On the other hand, we know that the nilpotent part of the closed-loop matrix is independent of the particular solution of $\operatorname{CGDARE}(\Sigma)$ considered. This means that all solutions of (28) have a closed-loop matrix that is non-singular;

- Given a solution $\Psi$ of (28), the null-space of $R_{0}+$ $B_{2}^{\mathrm{T}} \Psi B_{2}$ coincides with the null-space of $R_{0}$, since the null-space of $R_{0}+B_{2}^{\mathrm{T}} \Psi B_{2}$ does not depend on the particular solution of (28) and we know that the zero matrix is a solution of (28).

As a result of this discussion, it turns out that given a reference solution $X_{0}$ of $\operatorname{CGDARE}(\Sigma)$, the solution of $\operatorname{GDRE}(\Sigma)$ with terminal condition $X_{T}=P$ can be computed backward as follows:

1) For the first $v$ steps, i.e., from $t=T$ to $t=T-v, X_{t}$ is computed by iterating the $\operatorname{GDRE}(\Sigma)$ starting from the terminal condition $X_{T}=P$;

2) In the basis that isolates the nilpotent part of $A_{X}$, we have

$$
\Delta_{T-v}=\left[\begin{array}{cc}
O & O \\
O & \Psi_{T-v}
\end{array}\right]
$$

From $t=T-v-1$ to $t=0$, the solution of $\operatorname{GDRE}(\Sigma)$ can be found iterating the reduced order GDRE in (27) starting from the terminal condition $\Psi_{T-v}$.

Remark 5.1: The advantage of using the reduced-order generalised difference Riccati algebraic equation (27) consists in the fact that the closed-loop matrix of any solution of the associated generalised discrete Riccati algebraic equation is non-singular. Hence, when the reduced-order system is left invertible, the solution of the reduced-order generalised difference Riccati algebraic equation (27) can also be computed in closed-form, using the results in [6] or [4], which are adaptation to the discrete case of the techniques first 
presented in [3], [9], [5]. Indeed, consider a solution $\Psi$ of (28) with its non-singular closed-loop matrix $A \Psi$ and let $Y$ be the corresponding solution of the closed-loop Hermitian Stein equation

$$
A_{\Psi} Y A_{\Psi}^{\mathrm{T}}-Y+B_{2}\left(R_{0}+B_{2}^{\mathrm{T}} \Psi B_{2}\right)^{-1} B_{2}^{\mathrm{T}}=0 .
$$

The set of solutions of the extended symplectic difference equation for the reduced system is parameterised in terms of $K_{1}, K_{2} \in \mathbb{R}^{n \times n}$ as

$$
\left[\begin{array}{c}
X_{t} \\
\Lambda_{t} \\
\Omega_{t}
\end{array}\right]=\left[\begin{array}{c}
I_{n-v} \\
\Psi \\
-K_{\Psi}
\end{array}\right] A_{\Psi}^{t} K_{1}+\left[\begin{array}{c}
Y A_{\Psi}^{\mathrm{T}} \\
\left(\Psi Y-I_{n-v}\right) A_{\Psi}^{\mathrm{T}} \\
-K_{\star}
\end{array}\right]\left(A_{\Psi}^{\mathrm{T}}\right)^{T-t-1} K_{2},
$$

for $0 \leq t \leq T$. The values of the parameter matrices $K_{1}$ and $K_{2}$ can be computed so that the terminal condition satisfies $X_{T}=I_{n}$ and $\Lambda_{T}=\Psi_{T-v}$. Such values exist because $A_{\Psi}$ is non-singular, and are given by

$$
\begin{aligned}
& K_{1}=A_{\Psi}^{-T}\left(I_{n}-Y\left(\Psi-\Psi_{T-v}\right)\right) \\
& K_{2}=\Psi-\Psi_{T-v} .
\end{aligned}
$$

Then, the solution of (27) is given by $\Psi_{t}=\Lambda_{t} X_{t}^{-1}$.

\section{REFERENCES}

[1] H. Abou-Kandil, G. Freiling, V. Ionescu and G. Jank. Matrix Riccati Equations in Control and Systems Theory. Birkhäuser, Basel, 2003.

[2] A. Ferrante. On the structure of the solution of discrete-time algebraic Riccati equation with singular closed-loop matrix. IEEE Transactions on Automatic Control, AC-49(11):2049-2054, 2004.

[3] A. Ferrante, G. Marro, and L. Ntogramatzidis, "A parametrization of the solutions of the finite-horizon LQ problem with general cost and boundary conditions". Automatica, 41(8): 1359-1366, 2005.

[4] A. Ferrante and L. Ntogramatzidis, "Employing the algebraic Riccati equation for a parametrization of the solutions of the finite-horizon LQ problem: the discrete-time case". Systems \& Control Letters, 54:693703, 2005.

[5] A. Ferrante, and L. Ntogramatzidis, "A Unified Approach to the Finite-Horizon Linear Quadratic Optimal Control Problem". European Journal of Control, 13/5: 473-488, 2007.

[6] A. Ferrante, and L. Ntogramatzidis, "A unified approach to finitehorizon generalized LQ optimal control problems for discrete-time systems". Linear Algebra and Its Applications, 425(2-3):242-260, 2007.

[7] A. Ferrante, and H.K. Wimmer, "Order reduction of discrete-time algebraic Riccati equations with singular closed-loop matrix”. Operators and Matrices, 1(1):61-70, 2007.

[8] V. Ionescu, C. Oară, and M. Weiss. Generalized Riccati theory and robust control, a Popov function approach. Wiley, 1999.

[9] L. Ntogramatzidis, and G. Marro, "A parametrization of the solutions of the Hamiltonian system for stabilizable pairs". International Journal of Control, 78(7): 530-533, May 2005.

[10] D. Rappaport and L.M. Silverman. Structure and stability of discretetime optimal systems. IEEE Transactions on Automatic Control, AC16:227-233, 1971.

[11] A.A. Stoorvogel and A. Saberi. The discrete-time algebraic Riccati equation and linear matrix inequality. Linear Algebra and its Applications, 274:317-365, 1998. 